\title{
Kinetics of oxygen defect in YBCO
}

\section{S DATTAGUPTA and S K GHOSHAL}

School of Physical Sciences, Jawaharlal Nehru University, New Delhi 110067, India

\begin{abstract}
Monte-Carlo simulations are described using the Kawasaki model of oxygen (dis)ordering in the basal plane of $\mathrm{YBa}_{2} \mathrm{Cu}_{3} \mathrm{O}_{7 . \delta}(\mathrm{YBCO})$. In this model the presence or absence of an interstitial oxygen defect is mapped into a pseudo Ising spin $s=+1$ or -1 . We study the rate law and the relaxation time of the order parameter and most importantly, the diffusion of oxygen in the basal plane across the structural phase transition in this system. In a separate investigation we have carried out numerical analysis of a mean-field dynamical model (with Kawasaki kinetics) to examine phase ordering dynamics and pattern formation, for $\delta=0$.
\end{abstract}

Keywords. Structural transition in YBCO; Kawasaki kinetics; oxygen diffusion; phase ordering.

\section{Introduction}

Oxygen ordering in the $\mathrm{CuO}$ basal plane of $\mathrm{YBa}_{2} \mathrm{Cu}_{3} \mathrm{O}_{7-\delta}(\mathrm{YBCO})$ has been a subject of great contemporary interest (deFontaine et al 1987). When the stoichiometry $\delta$ equals zero the system has a superconducting transition around $90 \mathrm{~K}$. The transition temperature $T_{\mathrm{c}}(\delta)$ is found to decrease as $\delta$ increases from 0 and approach 0 as $\delta$ reaches the value 0.5 (Szpilka et al 1990). In addition to this interesting link between superconductivity and oxygen-stoichiometry, YBCO is also found to exhibit a structural transition from the tetragonal phase to the orthorhombic phase at a temperature $T_{\mathrm{s}}$ which is about $900 \mathrm{~K}$, for $\delta=0$. As oxygen ordering is also thought to give rise to the orthorhombicity of the crystal it is important to understand the mechanism of structural phase transition in YBCO (Dattagupta and Ghoshal 1993). In recent years a large number of analytical as well as numerical treatments involving a lattice gas/Ising model with three interaction parameters has been reported, in which the presence or the absence of oxygen atoms is modelled in terms of a pseudo spin $s=+1$ or - 1 (deFontaine et al 1987; Wille et al 1988; Berera et al 1989; Burmister and Wille 1989; Wille 1989; Szpilka et al 1990; Dattagupta and Ghoshal 1993). The tetragonal phase is then paramagnetic while the orthorhombic phase is antiferromagnetic with a nonzero staggered magnetization. $\mathrm{MC}$ simulation has been done using the Glauber (1963) (nonconserved spin flip) kinetics in order to reproduce the phase diagram of YBCO (Aukrust et al 1990; K hanna et al 1992). However, for a fixed $\delta$, the oxygen concentration may be held constant by adjusting the partial pressure of oxygen and hence the Kawasaki (1966) model that assumes spontaneous spin-exchanges corresponding to interchange of oxygen and vacancy on neighbouring sites, thus keeping the oxygen number fixed, is more appropriate. We should emphasize that although the oxygen number is conserved in the Kawasaki model, the relevant order parameter, which in the present case of antiferromagnetic'ordering is the staggered magnetization, is nonconserved.

While much attention has been focussed on static aspects such as order parameter variation with temperature, phase diagrams $\left(T_{\mathrm{s}} \mathrm{vs} \delta\right)$, etc, relatively fewer studies exist on kinetics of the anisotropic Ising model (Zhi-Xiong and Mahanti 1988; Aukrust et al 
1990; Choi et al 1990; Salmons and deFontaine 1990; Khanna et al 1992; Zhang and Catlow 1992; Fiig et al 1993). It is this aspect that is the main issue of interest in the present paper in which we present results on the order parameter relaxation when the system is either quenched or shock-heated across $T_{\mathrm{s}}$ as well as the relaxation time for the order parameter. We also study in detail the diffusive behaviour of oxygen in the $\mathrm{CuO}$ plane in the background of a rigid $\mathrm{Cu}$ lattice. The diffusion of oxygen determines the rate of oxygenation and hence the kinetics of ordering in the orthorhombic phase, leading to superconducting properties as well as formation of tweeds and textures (Salje 1993; Smenovskaya and Khachaturyan 1993).

Apart from the interest in high temperature superconductivity, the dynamics of nonequilibrium ordering processes is an interesting area of research in its own right (Gunton et al 1983). A theoretical explanation of these phenomena is extremely difficult due to the strong nonlinearities inherent in far-from-equilibrium problems. Layered high- $T_{c}$ superconductors of the YBCO type constitute a particularly interesting class of systems for studying such processes. Our aim here is to examine the nature of domain growth law in the $\mathrm{OI}(\delta=0)$ phase, following a mean field dynamical simulation scheme which is different from the MC approach of Poulsen et al (1991). We also study the form of the time-dependent structure factor which characterizes the evolving morphology and measure the length scales in different directions to check the directional dependence (if any) of the growth. Preliminary data for the growth law in different directions in the orthorhombic-I phase shows a $t^{1 / 2}$ behaviour, i.e. the so-called Lifshitz-Allen-Cahn growth law, in agreement with Poulsen et al (1991). The scaled structure factors in different directions collapse onto the same curve indicating that a single characteristic length scale is involved in the dynamical evolution (Ahluwallia et al, in preparation).

\section{Monte-Carlo study of oxygen diffusion in YBCO}

The anisotropic Ising model with three energy parameters $J_{\mathrm{nn}}, J_{\mathrm{nn}}^{\mathrm{B}}$ and $J_{\mathrm{nnn}}^{\mathrm{U}}$ introduced by deFontaine et al (1987) (figure 1) is described by the Hamiltonian

$$
H=J_{\mathrm{nn}} \sum_{i, j}^{\mathrm{nn}} s_{i} s_{j}+J_{\mathrm{nnn}}^{\mathrm{B}} \sum_{i, j}^{\mathrm{nn}(\mathrm{Cu})} s_{i} s_{j}+J_{\mathrm{nnn}}^{\mathrm{U}} \sum_{i, j}^{\mathrm{nnn}} s_{i} s_{j},
$$

where $\dot{s}_{i}$ is defined on the sites of a square lattice of the oxygen atom, $s_{i}=1$ specifying occupancy while $s_{i}==-1$ indicating vacancy, and $J_{\mathrm{nn}}$ is the nearest-neighbour (nn) interaction strength. Interactions, between a pair of oxygen atoms at next-nearestneighbour ( $\mathrm{nnn}$ ) sites are given by $J_{\mathrm{nnn}}^{\mathrm{B}}$ or $J_{\mathrm{fnn}}^{\mathrm{U}}$ depending on whether or not the pair is bridged by a copper atom. Our attention is presently directed onto the case $\delta=0$ that is known to yield a perfectly ordered antiferromagnetic structure, at $T=0$, corresponding to alternate oxygen-vacancy arrangement and hence, the appropriate order parameter is the staggered magnetization:

$$
Q=m_{a}-m_{b}=1 / N\left[\sum_{i \in a}\left\langle S_{i}^{a}\right\rangle-\sum_{i \in b}\left\langle S_{i}^{b}\right\rangle\right],
$$

where the indices $a$ and $b$ stand for the sublattice positions on the $a$ and $b$ axes (figure 1) of the basal plane, $N$ the total number of sites and the angular brackets $\langle\cdots\rangle$ indicate thermal averaging. 


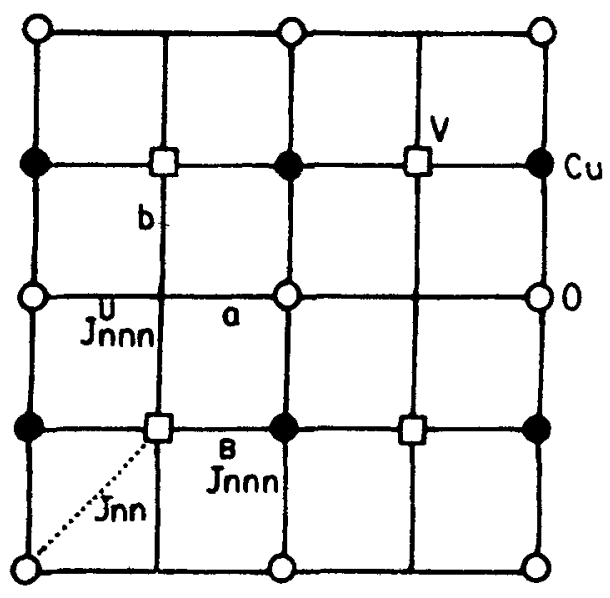

Figure 1. Two-dimensional model of the $\mathrm{YBa}_{2} \mathrm{Cu}_{3} \mathrm{O}_{7-\delta}$ basal-plane. Black circles represent copper, open circles denote oxygen and squares are vacancies. The two oxygen sublattices are denoted by $a$ and $b$.

We subject the system to standard Monte-Carlo simulations using the Metropolis scheme and the Kawasaki model wherein oxygen atoms are allowed to jump into vacant nearest neighbour sites on the lattice (Binder 1979; Ghoshal et al 1997). The change in energy associated with a pair of sites due to a particle-vacancy exchange forms the input in the Boltzmann factor, which measures the probability of an exchange. We have employed periodic boundary conditions and have eliminated possible size effects taking different sizes $L=32,64,128$ and 256 (Ghoshal et al 1997). Here $L$ is defined along the unit vector of the $\mathrm{Cu}$ unit cell and measured in units of the lattice constant.

\subsection{The relaxation time}

We turn our attention in this and the subsequent sections to the time-dependent behaviour of the system. Using the MC data points for Kawasaki kinetics we extract the relaxation time $\tau$, above and below $T_{s}$, from the definition:

$$
\tau=\sum_{t=0}^{t_{\max }}\left[\frac{Q(t)-Q(0)}{Q(0)-Q\left(t_{\max }\right)}\right],
$$

where $t_{\max }$ is the time taken for $Q(t)$ to attain its asymptotic value. That is, $Q\left(t_{\max }\right)=Q$ in equilibrium, at a given temperature. The plot of $\tau$ versus $T$ in figure 2 demonstrates the expected divergence near $T_{s}$, characteristic of the critical slowing down near a second order phase transition.

\subsection{The rate law}

In this subsection we present results on the order parameter evolution. For this, we start from the tetragonal phase, i.e. $Q(t=0)=0$ and quench below $T_{s}$ into the orthorhombic phase. The order parameter is expected to grow into a saturation value 


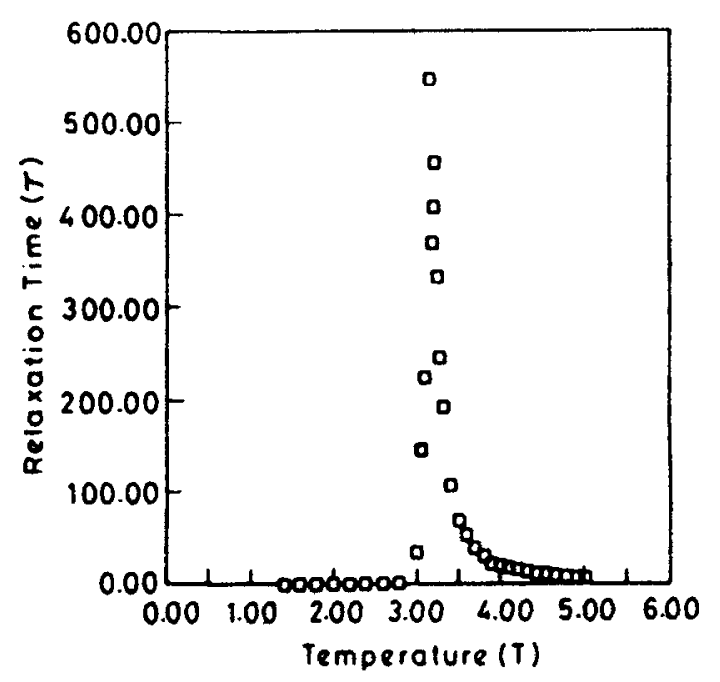

Figure 2. Relaxation time $(\tau)$ of the order parameter vs temperature $(T)$.

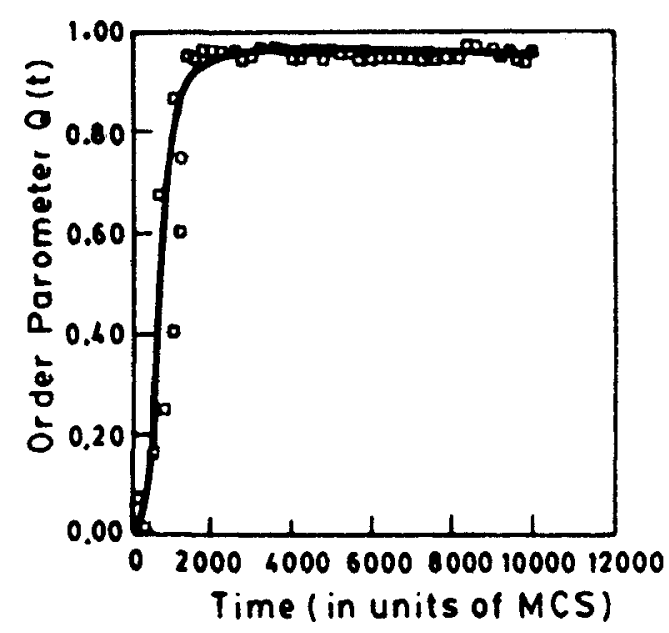

Figure 3. Growth of the order parameter $(Q)$ as a function of time $(t)$ as the system is quenched from $T \gg T_{\mathrm{s}}$ to $T=0.75 T_{\mathrm{s}}$; solid line: based on Kawasaki rate law in mean field theory (Dattagupta et al 1991; Dattagupta 1993) and squares: based on MC data.

which is higher the deeper we quench. The results are plotted in figure 3 for quenching temperature $0.75 T_{\mathrm{s}}$.

The Monte-Carlo data are also compared with analytical results obtained by us earlier using a certain mean field-like approximation (Dattagupta et al 1991; Dattagupta 1993) where we had considered the first moment of the master equation for the probability of spin configuration in the Kawasaki model and employed a random phase decoupling of correlation functions. The resultant equation of $Q(t)$ in terms of a certain scaled time reads:

$$
\frac{\mathrm{d}}{\mathrm{d} t} Q(t)=-\left(2 Q(t)-\left[1+Q^{2}(t)\right] \tan h\left[2 T_{\mathrm{s}} / T Q(t)\right]\right)
$$


The agreement with the data in figure 3 is excellent even though the present model is a short-ranged one. This surprising fact underscores how well the mean field theory works away from the critical region even if interactions are not long ranged, provided one uses a scaled temperature $T / T_{\mathrm{s}}$.

\subsection{Basal-plane diffusion}

As stated earlier, it is reasonable to assume that the copper atoms are rigidly fixed while studying oxygen diffusion in the basal plane. Diffusion occurs as the oxygen atom, aided by thermal fluctuations, is able to overcome a potential barrier and jump into a vacant site. Here we present data on the diffusion coefficient of oxygen using Kawasaki kinetics, in the tetragonal phase (and partly below $T_{\mathrm{s}}$ ) of YBCO. While in the completely disordered phase the diffusion is expected to be governed by the Arrhenius law, we are interested in studying its behaviour as one approaches $T_{\mathrm{s}}$. Near $T_{\mathrm{s}}$ there is a tendency for 'short-range' orthorhombic order and hence the diffusion is expected to be hindered.

For the study of diffusion we tag a single oxygen atom at the middle of the lattice and monitor its jump-displacement after each $50 \mathrm{MC}$ time steps, chosen in such a way that we can terminate the jumps before the particle hits the boundary. The diffusion coefficient $D$ is calculated from the following relation:

$$
D=\lim _{t \rightarrow \infty} 1 / t\left[\left\langle R^{2}(t)\right\rangle-\langle R(t)\rangle^{2}\right]
$$

where

$$
R(t)=\left\{(X(t)-X(0))^{2}+(Y(t)-Y(0))^{2}\right\}^{1 / 2},
$$

the angular brackets denoting equilibrium averages. In order to ensure that the asymptotic $(t \rightarrow \infty)$ limit is reached, we ignore all transient data for mean-square displacement and consider only those which depend linearly on the time $t$. From the plot of $R^{2}(t)$ vs $t$ (figure 4a), $D$ is estimated, for $\delta=0$. This computation is repeated for different temperatures. Needless to say, calculations become more time consuming as
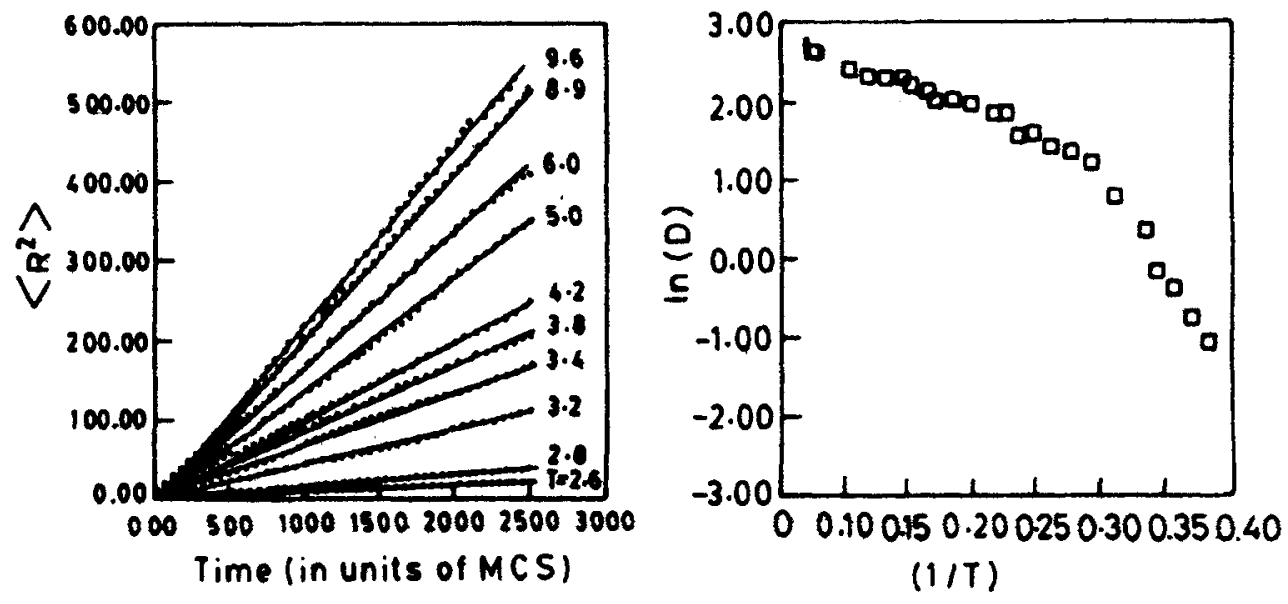

Figure 4. a. Mean square distance $\left(R^{2}\right)$ vs time $(t)$ in units of $25 \mathrm{MC}$ time steps, for $\delta=0$ and b. diffusion coefficient (D) vs $T^{-1}$ in a $\log$ plot for both $T>T_{\text {s }}$ and $T<T_{s}$, for $\delta=0$. 


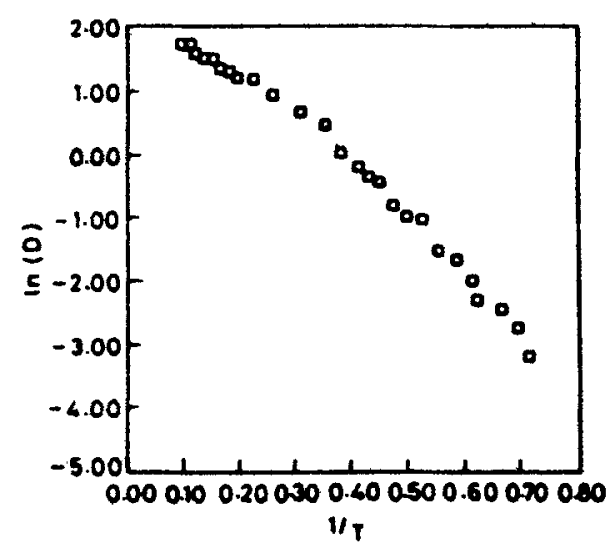

Figure 5. Diffusion coefficient (D) vs $T^{-1}$ in a $\log$ plot for both $T>T_{\mathrm{s}}$ and $T<T_{\mathrm{s}}$ for $\delta=0.5$.

$T_{\mathrm{s}}$ is approached. The extracted temperature dependence of $D(T)$ is plotted in figure $4 \mathrm{~b}$ in a $\ln (D)$ vs $1 / T$ graph which clearly indicates a departure from the Arrhenius behaviour at a temperature where there is a discernible change in slope. Therefore, the present technique yields a dynamic method for evaluating the temperature of phase change. Diffusion is exceedingly slow much below $T_{\mathrm{s}}$ and not amenable to computation. For $\delta \neq 0$, oxygen ordering in the orthorhombic phase is not simple antiferromagnetic. In fact, all sorts of complex phase diagrams have been reported in the literature for $0<\delta<0.5$, until for $\delta=0.5$ one finds double-chain (OII) formations (Szpilka et al 1990). While begging the issue of the nature of phase ordering for $0<\delta<0.5$ we can nevertheless estimate the transition temperature $T_{\mathrm{s}}$ by examining the diffusion data. Thus, we plot in figure 5 the temperature dependence of $D(T)$ for $\delta=0 \cdot 5$. The respective transition temperature $T_{\mathrm{s}}$ shows a fall in qualitative agreement with experiments (Cava et al 1987).

\section{Phase ordering dynamics in YBCO}

\subsection{Mean-field dynamical model}

We now formulate our mean-field dynamical model for phase ordering dynamics in YBCO, starting from (1). For Kawasaki(1966) spin exchanges between the site variables $s_{i} \mathrm{~S}$ and $s_{L_{i}} \mathrm{~s}$ (where $L_{i}$ refers to a neighbour of $i$ ) the relevant equation, obtained from the first moment of the master equation for the probability distribution of spin configurations, reads (Binder 1977):

$$
\begin{aligned}
2 \tau_{k} \frac{\partial}{\partial t}\left\langle s_{k}\right\rangle= & -q\left\langle s_{k}\right\rangle+\sum_{L_{k}}\left\langle s_{L_{k}}\right\rangle+\sum_{L_{k}}\left(1-\left\langle s_{k}\right\rangle\left\langle s_{L_{k}}\right\rangle\right) \tan h \frac{1}{T} \\
& \times\left[J_{\mathrm{nn}}\left[\sum_{L_{k}}\left\langle s_{j}\right\rangle-\sum_{\mathrm{nn}\left(L_{k}\right)}\left\langle s_{j}\right\rangle\right]+J_{\mathrm{nnn}}^{\mathrm{B}}\left[\sum_{\mathrm{nnn}(k)}^{\mathrm{Cu}}\left\langle s_{j}\right\rangle\right.\right. \\
& \left.\left.-\sum_{\operatorname{nnn}\left(L_{k}\right)}^{\mathrm{Cu}}\left\langle s_{j}\right\rangle\right]+J_{\mathrm{nnn}}^{\mathrm{U}}\left[\sum_{\operatorname{nnn}(k)}\left\langle s_{j}\right\rangle-\sum_{\operatorname{nnn}\left(L_{k}\right)}\left\langle s_{j}\right\rangle\right]\right] .
\end{aligned}
$$




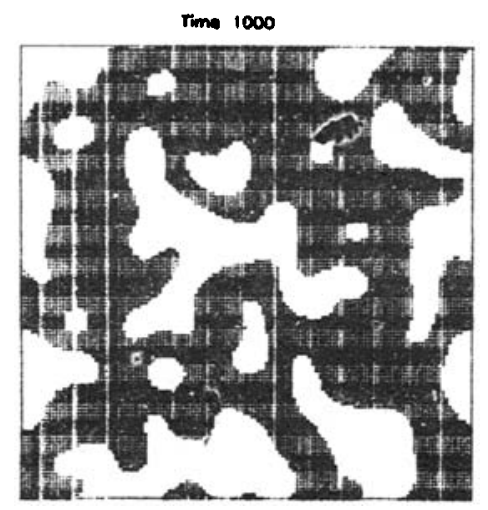

Figure 6. Temporal evolution of a disordered initial condition for our mean-field dynamical model. The initial condition for the oxygen vacancy field $\left\langle s_{k}\right\rangle$ consists of uniformly distributed random fluctuations of amplitude 0.05 around a zero background. Evolution picture is shown at time 1000 .

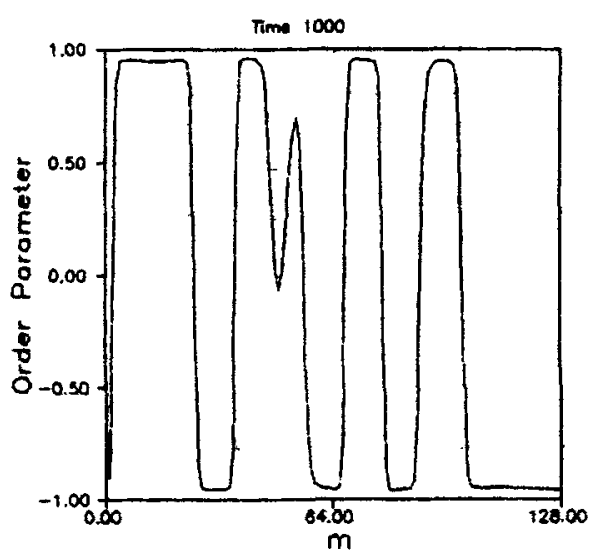

Figure 7. Order parameter profile for the temporal evolution shown in figure 6 . The solid line refers to the oxygen-vacancy field.

Numerical results from our simulations of (7) on an $N \times N$ lattice with periodic boundary conditions in both directions are presented here. A simple Euler discretization scheme is used with mesh size $\Delta t=0.01$ (the time $2 \tau_{k}$ has been rescaled into the time variable). Our parameter values are $T \simeq 0.5 T_{\mathrm{s}}$ and $J_{\mathrm{m}}=1 \cdot 0, J_{\mathrm{nnn}}^{\mathrm{B}}=-0.1$ and $J_{\mathrm{nn}}^{\mathrm{U}}=0.1$ (Wille et al 1988). The dynamics in the OI phase is charaeterized by a nonconserved order parameter (staggered magnetization). The corresponding saturation value of the order parameter is $Q=0.995$. The low temperature data are obtained by quenching the system from an initial disordered (paramagnetic) state to the low temperature ordered (antiferromagnetic) phase. Thus, we choose the initial condition for the oxygen vacancy field $\left(\left\langle S_{k}\right\rangle\right)$ as a uniformly distributed random fluctuation (of amplitude 0.05) about a zero background. This corresponds to a critical quench with equal amounts of oxygen and vacancy. Figure 6 shows the evolution of domains, starting from a disordered initial state, for $\delta=0$ and for $N=256$. The boundary which separates the region marked black from that which is not marked is distinguished 
where two neighbouring sites have the same spin, either +1 or -1 . Figure 7 shows the order parameter profiles for the evolution depicted in figure 6. The profiles are measured along a cross-section parallel to the horizontal axis and at the centre of the vertical axis.

\section{Conclusion}

In $\S 2$ we have presented results of MC study of Kawasaki kinetics of oxygen defects, based on a two-dimensional Ising model with parameters $J_{\mathrm{nn}}=1.0, J_{\mathrm{nnn}}^{\mathrm{B}}=-0.5$, $J_{\mathrm{nn}}^{\mathrm{U}}=+0.5$, following Wille et al (1988). Relaxation time of the order parameter evolution and the diffusion data are consistent with a continuous transition from the tetragonal to the orthorhombic phase (Zhang et al 1990).

In $\$ 3$ we have addressed a different issue, viz. phase ordering dynamics and pattern formation in YBCO. Here we have used the mean-field approximation and directly simulated the resultant dynamical equation from a disordered initial condition. The dynamics is characterized by algebraic growth law for nonconserved order parameter, at least in the OI phase. A detailed study of the growth laws and the form of the timedependent structure factor for different values of $\delta$ is in progress (Ahluwallia et al, in preparation). Finally, we stress that although our $2 d$ model captures the essential aspects of oxygen kinetics in $\mathrm{YBCO}$, the full $3 d$ problem has to be dealt with for a complete description of the ordering processes (Khachaturyan and Morris 1987).

\section{Acknowledgement}

The work on diffusion kinetics is based on collaboration with B Biswal while that on phase ordering is being carried out in collaboration with $\mathrm{R}$ Ahluwallia and S Puri.

\section{References}

Aukmust T, Novotny M A, Rixvold P A and Landau D P 1990 Phys. Rev. B41 8772

Berera A, Wille L T and deFontaine D 1989 J. Stat. Phys. 501245

Binder K 1977 Phys. Rev. B15 4425

Binder K 1979 Monte-Carlo methods in statistical physics (Berlin: Springer Verlag)

Burmister C P and Wille L T 1989 Phys. Rev. B40 8795

Cava R J, Batlogg B, Chen C H, Rietman E A, Zahurak S M and Werder D 1987 Nature 329423

Choi J S, Sarikaya M, Aksay I A and Kikuchi R 1990 Phys. Rev. B42 4244

Dattagupta S 1993 Physica A194 137

Dattagupta S and Ghoshal S K 1993 Solid State Commun. 88547

Dattagupta S, Heine V, Marais S and Salje E K H 1991 J. Phys.: Condens. Matter 32975

deFontaine D, Wille L T and Moss S C 1987 Phys. Rev. B36 5709

Fiig T, Andersen J V, Andersen N H, Lindgard P A, Mouritsen O G and Poulsen H F 1993 Physica C217 34

Ghoshal S K, Biswal B and Dattagupta S 1997 unpublished

Glauber R J 1963 J. Math. Phys. 4294

Gunton J D, San Miguel M and Sahni P S 1983 Phase transitions and critical phenomena (eds) C Domb and J L Lebowitz (New York: Academic Press) 8

Kawasaki K 1966 Phys. Rev. 145224

Khachaturyan A G and Morris J W 1987 Phys. Rev. Lett. 592776

Khanna R, Welberry T R and Ananthakrishna G 1992 Physica C197 57 
Poulsen H F, Andersen N H, Andersen J V, Bohr H and Mouritsen O G 1991 Nature 349594 and references therein

Salje E K H 1993 Phase transition in ferroelastic and co-elastic crystals (Cambridge: Cambridge University Press)

Salmons E and deFontaine D 1990 Phys. Rev. B41 11159

Smenovskaya S and Khachaturyan A G 1993 Physica D66 205

Szpilka A M, Glasser M L, Mattis D C and Mattis M P 1990 Phase Transitions 22185

Wille L T 1989 Phys. Rev. B40 6931

Wille L T, Berera A and deFontaine D 1988 Phys. Rev. Lett. 601065

Zhang J X, Lin G M, Zeng W G, Liang K F, Lin Z C, Siu G G, Stokes M J and Fung P C W 1990 Supercond. Sci. Technol. 3113

Zhang X and Catlow C R A 1992 Phys. Rev. B46 457

Zhi-Xiong C and Mahanti S D 1988 Solid State Commun. 67287 